\title{
Multi-material topology optimization of laminated composite beams with eigenfrequency constraints
}

\author{
Blasques, José Pedro Albergaria Amaral
}

Published in:

Composite Structures

Link to article, DOI:

10.1016/j.compstruct.2013.12.021

Publication date:

2014

Link back to DTU Orbit

Citation (APA):

Blasques, J. P. A. A. (2014). Multi-material topology optimization of laminated composite beams with eigenfrequency constraints. Composite Structures, 111, 45-55. https://doi.org/10.1016/j.compstruct.2013.12.021

\section{General rights}

Copyright and moral rights for the publications made accessible in the public portal are retained by the authors and/or other copyright owners and it is a condition of accessing publications that users recognise and abide by the legal requirements associated with these rights.

- Users may download and print one copy of any publication from the public portal for the purpose of private study or research.

- You may not further distribute the material or use it for any profit-making activity or commercial gain

- You may freely distribute the URL identifying the publication in the public portal

If you believe that this document breaches copyright please contact us providing details, and we will remove access to the work immediately and investigate your claim. 


\title{
Multi-material topology optimization of laminated composite beams with eigenfrequency constraints
}

\author{
José Pedro Blasques*
}

Department of Wind Energy, Technical University of Denmark, Frederiksborgvej 399, Building 114, 4000 Roskilde, Denmark

\begin{abstract}
This paper describes a methodology for simultaneous topology and material optimization in optimal design of laminated composite beams with eigenfrequency constraints. The structural response is analyzed using beam finite elements. The beam sectional properties are evaluated using a finite element based cross section analysis tool which is able to account for effects stemming from material anisotropy and inhomogeneity in sections of arbitrary geometry. The optimization is performed within a multi-material topology optimization framework where the continuous design variables represent the volume fractions of different candidate materials at each point in the cross section. An approach based on the Kreisselmeier-Steinhauser function is proposed to deal with the non-differentiability issues typically encountered when dealing with eigenfrequency constraints. The framework is applied to the optimal design of a laminated composite cantilever beam with constant cross section. Solutions are presented for problems dealing with the maximization of the minimum eigenfrequency and maximization of the gap between consecutive eigenfrequencies with constraints on the weight and shear center position. The results suggest that the devised methodology is suitable for simultaneous optimization of the cross section topology and material properties in design of beams with eigenfrequency constraints.
\end{abstract}

Keywords: Beams, Cross section analysis, Multi-material topology optimization, Eigenfrequency constraints, Kreisselmeier-Steinhauser function

\section{Introduction}

A typical objective in the design of flexible structures subjected to dynamic loads concerns the maximization of the minimum eigenfrequency or the maximization of the gap between consecutive eigenfrequencies. From the many different methodologies proposed in the literature, topology optimization techniques have proved a promising alternative. Diaz and Kikuchi [1] and Ma et al. [2] presented results for structural topology optimization of two-dimensional structures. Pedersen [3] and Du and Olhoff [4] addressed the problem concerning the control of the dynamic properties of plates. Luo and Gea [5] and Gea and Luo [6] presented a strategy for optimizing the location and orientation of stiffeners for eigenfrequency placement design of shell structures. Furthermore, Stegmann and Lund [7] and Pedersen [8] have presented solutions for the maximization of the minimum eigenfrequency design of laminated composite plates. The optimal design of beams with eigenfrequency constraints, however, has mostly concerned two dimensional problems addressing only the optimization of the cross section dimensions along the beam length (see, e.g., Olhoff [9] and Bendsøe and Olhoff [10]).

An extension of the computational framework suggested by Blasques and Stolpe [11] combining a high-fidelity beam model

\footnotetext{
${ }^{*}$ Corresponding author. Phone: +4560608606

Email address: jpbl@dtu.dk (José Pedro Blasques*)
}

and multi-material topology optimization techniques, is presented here to include eigenfrequency constraints. Preliminary results are presented in which the cross section topology and laminate properties of prismatic cantilevered laminated composite beams are optimized simultaneously. It is shown that the framework is suitable for eigenfrequency tailoring of a general class of beam-like structures. Potential applications include aeroelastic optimization of wind turbine blades for mitigation of aeroelastic instabilities, among other. To the author's best knowledge no previous publication addresses the simultaneous topology and material optimization of beam cross sections with eigenfrequency constraints as presented here.

The proposed framework relies on a high-fidelity beam finite element model for the analysis of the structural response. These type of modelling approach allows for a computationally inexpensive representation of three dimensional beam-like structures. The global response of the beam - e.g., compliance and eigenfrequencies - can be determined with great accuracy using a model which is computationally much less costly than its three-dimensional shell or solid finite element counterparts. This capability has been exploited in computationally intensive applications, e.g., wind turbine aeroelastic simulation tools (see, e.g., Larsen and Hansen [12]). The generation of the beam model is divided in two parts. The first and most challenging part concerns the solution of a two-dimensional problem dealing with the determination of the cross section stiffness and mass properties. In the second part, the previ- 
ously computed cross section properties are integrated along the beam length to obtain the beam finite element stiffness and mass matrices. The sectional properties are analyzed here using the BEam Cross section Analysis Software (BECAS), an open-source implementation by Blasques and Lazarov [13] of the original theory by Giavotto et al. [14]. BECAS is a finite element based tool which is able to account for the effects of material anisotropy and inhomogeneity in the analysis of the stiffness and mass properties of beam sections of arbitrary geometry. The reader is referred to Jung et al. [15], Volovoi et al. [16], and the comprehensive work by Hodges [17] for a review on different beam modelling techniques.

In this context, the optimal design problem concerns the distribution of a limited amount of different materials within a design domain represented here by the cross section finite element mesh. A change in the material distribution in the cross section results in a consequent change of its stiffness and mass properties and in turn, of the structural response of the beam. This optimal design problem is solved using the multi-material topology optimization framework presented by Blasques and Stolpe [11], Hvejsel and Lund [18], and Hvejsel et al. [19]. The framework is based on the principles of topology optimization (see, e.g., Bendsøe and Sigmund [20]) and relies on extensions to include multiple anisotropic materials of the Solid Isotropic Material with Penalization (SIMP) material interpolation technique (Bendsøe and Kikuchi [21] and Rozvany et al. [22]), and the density filtering scheme by Bruns and Tortorelli [23]. This approach is a variation of the so-called discrete material optimization technique originally presented by Lund and Stegmann [24] and Stegmann and Lund [7] and applied to the optimal design of laminated composite shell structures.

A common issue when dealing with eigenfrequency constraints concerns the fact that the order of the eigenfrequencies may change throughout the optimization procedure. This will in turn lead to non-differentiability and consequently to a nonrobust convergence behaviour of methods for smooth optimization, namely, gradient-based methods. A typical approach to mitigate these effects consists of applying the so-called bound formulation (see, e.g., Bendsøe and Sigmund [20]). An alternative approach is proposed here using the KreisselmeierSteinhauser (KS) function (Kreisselmeier and Steinhauser [25]) to approximate the maximum and minimum values of groups of eigenfrequencies. The KS function is a continuously differentiable envelope function which approximates the maximum or minimum of a set of functions. The functions should be continuous but need not be continuously differentiable. The aim is to try to improve the convergence behaviour by rewriting the eigenfrequency constraints to take advantage of the mathematical properties of the KS function. The mathematical properties of the KS function have been discussed by Raspanti et al. [26]. Moreover, it has been used in similar optimal structural design contexts as a constraint aggregation function by, e.g., Martins et al. [27] and Maute et al. [28].

The paper is organized as follows. The beam finite element structural model is briefly described in Section 2. The multi-material topology optimization framework and problem formulations are described in Section 3, where the KS func- tion is also presented. The gradients or sensitivities for each of the objective functions and constraints are presented in Section 4. Section 5 describes the setup of the numerical experiments, presents the optimized cross section designs, and discusses the results. Finally, the most important conclusions of the work presented in this paper are summarized in Section 6.

\section{Structural model}

The structural response of the beam is analyzed based on the beam finite element model presented by Blasques and Stolpe [11]. The model is extended here for the analysis of the beam eigenfrequencies and eigenmodes.

When using beam models it is assumed that the original beam structure is represented by a reference line along the length of the beam going through the reference points of a given number of representative cross sections. The two steps involved in the generation of the beam model are discussed next. The first step concerns the evaluation of the cross section stiffness and mass properties as discussed in Section 2.1. The second part concerns the integration of these properties to generate the beam finite elements. The latter is addressed in Section 2.2 where the derivation of the beam finite element stiffness and mass matrices is presented along with the equations of motion for the analysis of the dynamic response of the beam.

\subsection{Cross section analysis}

For a linear elastic beam there exists a linear relation between the cross section generalized forces $\mathbf{T}$ and moments $\mathbf{M}$ in $\boldsymbol{\theta}=\left[\mathbf{T}^{T} \mathbf{M}^{T}\right]^{T}$, and the resulting strains $\boldsymbol{\tau}$ and curvatures $\boldsymbol{\kappa}$ in $\boldsymbol{\psi}=\left[\boldsymbol{\tau}^{T} \boldsymbol{\kappa}^{T}\right]^{T}$ (see Figure 1). This relation is given in its stiffness form as $\mathbf{K}_{s} \boldsymbol{\psi}=\boldsymbol{\theta}$, where $\mathbf{K}_{s}$ is the $6 \times 6$ cross section stiffness matrix. In the most general case, considering material anisotropy and inhomogeneity, all the 21 stiffness parameters in $\mathbf{K}_{s}$ may be required to describe the deformation of the cross section. In the current research, the entries of $\mathbf{K}_{s}$ are determined using the BEam Cross section Analysis Software (BECAS), an implementation by Blasques and Lazarov [13] of the theory by Giavotto et al. [14]. The formulation relies on a finite element discretization of the cross section to approximate the cross section in-plane and out-of-plane deformation or warping. BECAS is able to estimate the stiffness properties of beam sections with arbitrary geometry and correctly account for the effects stemming from material anisotropy and inhomogeneity. A brief outline of the theory underlying the determination of $\mathbf{K}_{s}$ is presented here. The reader is referred to Blasques and Stolpe [11] for more details on the derivation and notation.

The determination of $\mathbf{K}_{s}$ entails the solution to a twodimensional problem associated with the determination of three-dimensional deformation of the cross section. The solution is obtained from the cross section equilibrium equations given by the following system of linear equations

$$
\mathbf{K W}=\mathbf{F}
$$

where he coefficients in matrix $\mathbf{K}$ are associated with the stiffness of the cross section. Furthermore, the solution matrix $\mathbf{W}$ 
contains the cross section rigid body motions $\psi$ and the three dimensional warping displacements $\mathbf{u}$. Finally, the load array $\mathbf{F}$ is associated with a series of unit load vectors $\boldsymbol{\theta}$. The solution $\mathbf{W}$ from (1) is subsequently used in the determination of the cross section compliance matrix $\mathbf{F}_{s}$ defined as

$$
\mathbf{F}_{s}=\mathbf{W}^{T} \mathbf{G W}
$$

where the coefficient matrix $\mathbf{G}$ is defined in Blasques and Stolpe [11]. For most practical applications, and in all cases considered in this paper, $\mathbf{F}_{s}$ is symmetric positive definite. Hence, the cross section stiffness matrix is consequently obtained from $\mathbf{K}_{s}=\mathbf{F}_{s}^{-1}$.

The analysis of the cross section mass properties is relatively simpler. The $6 \times 6$ cross section mass matrix $\mathbf{M}_{s}$ relates the linear and angular velocities in $\phi$ to the generalized inertial linear and angular momentum in $\gamma$ through $\phi=\mathbf{M}_{s} \gamma$. According to Hodges [17], the coefficients of $\mathbf{M}_{s}$ are

$$
\mathbf{M}_{s}=\left[\begin{array}{cccccc}
m & 0 & 0 & 0 & 0 & -m y_{m} \\
0 & m & 0 & 0 & 0 & m x_{m} \\
0 & 0 & m & m y_{m} & -m x_{m} & 0 \\
0 & 0 & m y_{m} & I_{x x} & -I_{x y} & 0 \\
0 & 0 & -m x_{m} & -I_{x y} & I_{y y} & 0 \\
-m y_{m} & m x_{m} & 0 & 0 & 0 & I_{x x}+I_{y y}
\end{array}\right]
$$

where $m$ is the mass per unit length, $I_{x x}$ and $I_{y y}$ are the moment of inertia with respect to $x$ and $y$, respectively, and $I_{x y}$ is the product of inertia. The off-diagonal terms are due to the offset between the position of the cross section reference center and the mass center $\mathbf{m}_{c}=\left(x_{m}, y_{m}\right)$. Here the reference center is defined as the point through which the reference line goes through and is coincident with the beam finite element discretization. All of the terms in $\mathbf{M}_{s}$ are determined through integration of the mass properties in the cross section finite element mesh.

\subsection{Beam finite element analysis}

The finite element form of the beam structural eigenvalue problem is (cf. Bathe [29])

$$
\left(\widehat{\mathbf{K}}-\omega_{f}^{2} \widehat{\mathbf{M}}\right) \widehat{\mathbf{v}}_{f}=0, \forall f=1, \ldots, n_{d}
$$

where $n_{d}$ is the number of degrees of freedom associated with the finite element stiffness and mass matrices, $\widehat{\mathbf{K}}$ and $\widehat{\mathbf{M}}$, respectively. The problem above yields the eigenfrequencies $\omega=$ $\left\{\omega_{1}, \ldots, \omega_{n_{d}}\right\}$ associated with the eigenvectors $\widehat{\mathbf{V}}=\left\{\widehat{\mathbf{v}}_{1}, \ldots, \widehat{\mathbf{v}}_{n_{d}}\right\}$. It is assumed that the eigenfrequencies in $\omega$ are given in ascending order of magnitude, i.e., $\omega_{1} \leq \omega_{2} \leq \ldots \leq \omega_{n_{d}}$.

The global beam stiffness matrix $\widehat{\mathbf{K}}$ is defined as

$$
\widehat{\mathbf{K}}=\sum_{b=1}^{n_{b}} \widehat{\mathbf{K}}_{b}=\sum_{b=1}^{n_{b}} \int_{0}^{L_{b}} \widehat{\mathbf{B}}_{b}^{T} \mathbf{K}_{s} \widehat{\mathbf{B}}_{b} \mathrm{~d} z
$$

where $n_{b}$ is the number of elements in the beam finite element assemblage, and $L_{b}$ is the length of element $b$. The summation refers to the typical finite element assembly. The beam finite element stiffness matrix $\widehat{\mathbf{K}}_{b}$ for element $b$ is given in function of $\widehat{\mathbf{B}}_{b}=\mathcal{B}\left(\widehat{\mathbf{N}}_{b}\right)$ where $\mathcal{B}$ is the strain-displacement relation which is a function of $\widehat{\mathbf{N}}_{b}$, the finite element shape function matrix. The cross section stiffness matrix $\mathbf{K}_{s}$ has been defined in the previous section. The beam global finite element mass matrix $\widehat{\mathbf{M}}$ is defined as

$$
\widehat{\mathbf{M}}=\sum_{b=1}^{n_{b}} \widehat{\mathbf{M}}_{b}=\sum_{b=1}^{n_{b}} \int_{0}^{L_{b}} \widehat{\mathbf{N}}_{b}^{T} \mathbf{M}_{s} \widehat{\mathbf{N}}_{b} \mathrm{~d} z
$$

where $\widehat{\mathbf{M}}_{b}$ is the beam finite element mass matrix for element $b$ and $\mathbf{M}_{s}$ is the cross section mass matrix defined in (3).

\section{Optimization model}

The optimal design problem is formulated based on the multi-material topology optimization framework presented by Blasques and Stolpe [11]. The aim is to determine the optimal distribution of a predefined set of candidate materials at each point of the beam cross section. The design requirements entail the maximization or minimization of specific eigenfrequencies of the beam.

A brief outline of the optimization framework is presented in Section 3.1. The optimal design problem formulation considered in this paper is subsequently presented in Section 3.2.

\subsection{Multi-material topology optimization}

It is assumed that a set of $n_{c}$ candidate materials has been defined. The candidate materials may be anisotropic or even the same anisotropic material oriented in different directions. A candidate material $m$ is defined by its constitutive matrix $\overline{\mathbf{Q}}_{m}$ and density $\bar{\varrho}_{m}$. An extension of the SIMP material interpolation model (Bendsøe and Kikuchi [21], and Rozvany and Zhou [22]) to multiple anisotropic materials is used. Hence, the material constitutive matrix $\mathbf{Q}_{e}$ at element $e$ is defined as

$$
\mathbf{Q}_{e}(\boldsymbol{\rho})=\sum_{m=1}^{n_{c}} \widetilde{\rho}_{e m}^{p}(\boldsymbol{\rho}) \overline{\mathbf{Q}}_{m} \quad, \quad \forall e=1, \ldots, n_{e}
$$

where $n_{e}$ is the number of elements in the cross section finite element mesh, $\widetilde{\rho}_{e m}$ is the filtered volume fraction of material $m$ at element $e$ as described later in this section, and $p$ is a penalization parameter. The volume fractions $\rho=\left\{\rho_{e m} \in \mathbb{R}\right.$ $\left.\mid e \in\left\{1, \ldots, n_{e}\right\}, \quad m \in\left\{1, \ldots, n_{c}\right\}\right\}$ are the design variables of the optimization problem. It is assumed that the design variables vary continuously between their bounds, i.e., $0 \leq \rho_{e m} \leq 1$, $\forall e=1, \ldots, n_{e}, \forall m=1, \ldots, n_{c}$. Furthermore, $\mathbf{Q}_{e}$ is constant within each cross section finite element. Finally, the density $\varrho_{e}$ at element $e$ is similarly defined as

$$
\varrho_{e}(\boldsymbol{\rho})=\sum_{m=1}^{n_{c}} \widetilde{\rho}_{e m}(\boldsymbol{\rho}) \bar{\varrho}_{m} \quad, \forall e=1, \ldots, n_{e}
$$

where the penalty parameter is not included.

The role of $p$ in (7) is to penalize intermediate material densities or volume fractions. As the magnitude of $p$ increases the design variables are pushed towards their bounds. The penalized problem, however, may have a large number of local minima. A continuation approach is therefore employed to increase the possibility of obtaining a good design (see Sigmund and Petersson [30], Borrval and Petersson [31], and Hvejsel and Lund 
[18]). The practical use of the penalty parameter $p$ within the continuation approach is further discussed in Section 5.2.

In the typical SIMP formulation for two phase problems an increase in the volume fraction of one material corresponds to a decrease in the volume fraction of the second material. The same effect is achieved here through the inclusion of the following set of $n_{e}$ linear constraints in the optimal design problem formulation

$$
\sum_{m=1}^{n_{c}} \rho_{e m}=1 \quad, \quad \forall e=1, \ldots, n_{e}
$$

Hvejsel and Lund [18] and Hvejsel et al. [19] studied the parametrization presented above in great detail and compared it with other equivalent techniques. This parameterization is a generalization of the SIMP approach and typical topology optimization techniques and formulations are therefore directly extendible to the multi-material case (e.g., the density filtering technique presented next). The potentially large number of additional linear constraints introduced in (8) can be formulated as sparse constraints which are easily handled by modern and robust gradient based algorithms like sNOPT by Gill et al. [32]. Moreover, it is possible to use this linear constraints to create relations between the different phases and locally control its distribution. Recent work by Sørensen and Lund [33] exploits these possibilities to include manufacturing constraints in the design of laminated composite shell structures.

Common issues in density based topology optimization problems concern the appearance of checkerboard patterns and the dependency of the results on the resolution of the finite element mesh (Sigmund [34]). The extension presented by Blasques and Stolpe [11] of the density filtering technique by Bruns and Tortorelli [23] is employed here to address these issues. In this approach, the volume fractions at an element are a function of the volume fractions of the surrounding elements within a given distance $f_{r}$ (the filter radius) and the element itself. The filtered volume fractions are defined as $\widetilde{\rho}_{e m}=\widetilde{\rho}_{\text {em }}\left(\rho_{\text {em }}\right)$, where a linear support function (cf. Bruns and Tortorelli [23] and Bourdin [35]) is used to weight the volume fractions.

Finally, the design variables $\rho$ enter the structural model through the material constitutive matrix $\mathbf{Q}_{e}=\mathbf{Q}_{e}(\rho)$ and densities $\varrho_{e}=\varrho_{e}(\rho)$. The material constitutive matrix $\mathbf{Q}_{e}(\rho)$ is required for the determination of the cross section coefficient matrices in (1) and (2) such that $\mathbf{K}=\mathbf{K}(\boldsymbol{\rho})$ and $\mathbf{G}=\mathbf{G}(\boldsymbol{\rho})$, respectively. Consequently, the cross section stiffness matrix is defined such that $\mathbf{K}_{s}=\mathbf{K}_{s}(\boldsymbol{\rho})$. The material densities $\varrho_{e}(\boldsymbol{\rho})$ are required in the evaluation of the cross section mass matrix in (3) such that $\mathbf{M}_{s}=\mathbf{M}_{s}(\boldsymbol{\rho})$. At the beam finite element level, $\widehat{\mathbf{K}}=\widehat{\mathbf{K}}(\rho)$ and $\widehat{\mathbf{M}}=\widehat{\mathbf{M}}(\rho)$.

\subsection{Problem formulation}

The eigenfrequencies of the beam $\omega(\rho)$ are introduced in the optimal design problem through the Kreisselmeier-Steinhauser (KS) function (cf. Kreisselmeier and Steinhauser [25]). The $\mathrm{KS}$ function is a differentiable envelope function which gives a conservative representation of the maximum or minimum of a set of functions (Raspanti et al. [26]). It is used here to approximate the maximum or minimum of a group of eigenfrequencies. The derivation presented next is for the form of KS approximating the minimum of a function - hereby denoted $\underline{\operatorname{KS}}(\rho)$. Hence, assume that $\underline{\omega}=\left\{\underline{\omega}_{1}, \ldots, \underline{\omega}_{n_{g}}\right\}$ is a subset of the eigenfrequencies $\omega=\left\{\omega_{1}, \ldots, \omega_{n_{d}}\right\}$ obtained from (4) whose minimum we wish to approximate. Furthermore, it is also assumed that the $n_{g}$ eigenfrequencies in $\underline{\omega}$ are ordered such that $\underline{\omega}_{1} \leq \ldots \leq \underline{\omega}_{n_{g}}$. In this case, $\underline{\operatorname{KS}}(\rho)$, is defined as

$$
\underline{\mathrm{KS}}(\boldsymbol{\rho})=-\frac{1}{\beta_{s}} \ln \left[\sum_{g=1}^{n_{g}} e^{-\beta_{s} \underline{\omega}_{g}(\boldsymbol{\rho})}\right]
$$

where the parameter $\beta_{s} \geq 1$ is such that the function $\underline{\operatorname{KS}}(\rho)$ will tend to the minimum of $\underline{\omega}$ as $\beta_{s}$ increases. In order to reduce numerical difficulties $\underline{\operatorname{KS}}(\boldsymbol{\rho})$ can be rewritten as

$$
\underline{\mathrm{KS}}(\boldsymbol{\rho})=\underline{\omega}_{n_{g}}(\boldsymbol{\rho})-\frac{1}{\beta_{s}} \ln \left[\sum_{g=1}^{n_{g}} e^{-\beta_{s}\left(\underline{\omega}_{g}(\boldsymbol{\rho})-\underline{\omega}_{g}(\boldsymbol{\rho})\right)}\right]
$$

The function $\overline{\mathrm{KS}}(\rho)$ approximating the maximum of a group of eigenfrequencies $\bar{\omega}$ is easily obtained from the expressions above.

The position of the shear center $\mathbf{s}_{c}(\boldsymbol{\rho})=\left(x_{s}(\boldsymbol{\rho}), y_{s}(\boldsymbol{\rho})\right)$ is defined in function of the entries $F_{s, i j}$ of the compliance matrix $\mathbf{F}_{s}(\boldsymbol{\rho})$ as (cf. Hodges [17])

$$
x_{s}(\boldsymbol{\rho})=-\frac{F_{s, 62}(\rho)}{F_{s, 66}(\rho)}, \quad y_{s}(\boldsymbol{\rho})=\frac{F_{s, 61}(\rho)}{F_{s, 66}(\rho)}
$$

where it is assumed that the component relating to the coupling between bending and torsion is nil. The shear center is defined such that a transverse load applied at the shear center will not induce a torsional moment.

The reference optimal design problem formulation (problem P1-1) concerns the maximization of the gap $\underline{\operatorname{KS}}(\underline{\omega}(\rho))-$ $\overline{\mathrm{KS}}(\bar{\omega}(\rho))$ between the minimum and maximum of the two groups of eigenfrequencies $\underline{\omega}(\rho)$ and $\bar{\omega}(\rho)$, respectively. The problem P1-1 including constraints on the shear center position, and total weight of the beam is formulated as

$$
\begin{array}{cl}
\underset{\rho \in \mathbb{R}^{n_{e} x_{c}}}{\operatorname{maximize}} & \underline{\mathrm{KS}} \underline{\omega}(\boldsymbol{\rho}))-\overline{\mathrm{KS}}(\overline{\boldsymbol{\omega}}(\boldsymbol{\rho})) \\
\text { subject to } & \underline{\mathbf{s}} \leq \mathbf{s}_{c}(\boldsymbol{\rho}) \leq \overline{\mathbf{s}} \\
& w(\boldsymbol{\rho})=\bar{w} \\
& \sum_{m=1}^{n_{c}} \rho_{e m}=1, \forall e=1, \ldots, n_{e} \\
& 0 \leq \rho_{e m} \leq 1, \forall e=1, \ldots, n_{e}, \\
& \forall m=1, \ldots, n_{c}
\end{array}
$$

where the parameters $\bar{w}$ and, $\overline{\mathbf{s}}$ and $\underline{\mathbf{s}}$ are the constraint values for the weight and shear center position, respectively. An equality constraint is used for the weight in order to avoid designs where only the material with lowest weight exists.

Alternative problem formulations can be obtained by rearranging the objective functions and constraints in $\mathrm{P} 1-1$. The 
following problem formulations have also been considered in this paper. Problem formulation P1-2 concerns the maximization of the gap between two groups of eigenfrequencies with constraints on the weight. Problem formulation P1-3 concerns the maximization of the minimum eigenfrequency with a constraint on the weight. Finally, problem formulation P1-4 concerns the maximization of the minimum eigenfrequency subject to constraints on weight and shear center position. Numerical results are presented in Section 5 for each of the problem formulation mentioned before.

The aim in this paper is to present proofs-of-concept for later research, namely, in the field of wind turbine blade design. Tailoring the magnitude of certain eigenfrequencies and controlling the relative position of the shear and mass center positions it is possible, e.g., to tailor the aeroelastic behaviour of the blade to mitigate the risk of dynamic instabilities (see, e.g., Hansen [36]).

\section{Sensitivities}

The analytical functions yielding the sensitivities of the beam eigenfrequencies and KS function are derived in this section. The gradients of the cross section stiffness matrix and shear center position have been derived in Blasques and Stolpe [11]. Hence, only a brief outline is presented here.

\subsection{Beam stiffness and mass matrix}

The sensitivities of the global beam finite element stiffness matrix $\widehat{\mathbf{K}}$ are obtained through differentiation of (5) to yield

$$
\frac{\partial \widehat{\mathbf{K}}(\boldsymbol{\rho})}{\partial \rho_{e m}}=\sum_{b=1}^{n_{b}} \int_{0}^{L_{b}} \widehat{\mathbf{B}}_{b}^{T} \frac{\partial \mathbf{K}_{s}(\boldsymbol{\rho})}{\partial \rho_{e m}} \widehat{\mathbf{B}}_{b} \mathrm{~d} z
$$

The gradient of the cross section stiffness matrix $\mathbf{K}_{s}$ is described in Section 4.2. The gradients of the global beam finite element mass matrix $\widehat{\mathbf{M}}(\rho)$ are obtained through differentiation of (6) and defined as

$$
\frac{\partial \widehat{\mathbf{M}}(\boldsymbol{\rho})}{\partial \rho_{e m}}=\sum_{b=1}^{n_{b}} \int_{0}^{L_{b}} \widehat{\mathbf{N}}_{b}^{T} \frac{\partial \mathbf{M}_{s}(\boldsymbol{\rho})}{\partial \rho_{e m}} \widehat{\mathbf{N}}_{b} \mathrm{~d} z
$$

\subsection{Cross section stiffness and mass matrix}

The sensitivities of the cross section stiffness matrix $\mathbf{K}_{s}(\boldsymbol{\rho})$ with respect to $\rho_{e m}$ are defined in function of the cross section compliance matrix $\mathbf{F}_{s}(\boldsymbol{\rho})$ as

$$
\frac{\partial \mathbf{K}_{s}(\boldsymbol{\rho})}{\partial \rho_{e m}}=\frac{\partial \mathbf{F}_{s}^{-1}(\boldsymbol{\rho})}{\partial \rho_{e m}}=-\mathbf{K}_{s}(\boldsymbol{\rho}) \frac{\partial \mathbf{F}_{s}(\boldsymbol{\rho})}{\partial \rho_{e m}} \mathbf{K}_{s}(\boldsymbol{\rho})
$$

The gradients of the cross section compliance matrix are obtained through differentiation of (2) which yields

$$
\begin{aligned}
\frac{\partial \mathbf{F}_{s}(\boldsymbol{\rho})}{\partial \rho_{e m}}= & -\mathbf{W}^{T}(\boldsymbol{\rho}) \frac{\partial \mathbf{K}^{T}(\boldsymbol{\rho})}{\partial \rho_{e m}} \mathbf{V}(\boldsymbol{\rho})+ \\
& \mathbf{W}^{T}(\boldsymbol{\rho}) \frac{\partial \mathbf{G}(\boldsymbol{\rho})}{\partial \rho_{e m}} \mathbf{W}(\boldsymbol{\rho})-\mathbf{V}^{T}(\boldsymbol{\rho}) \frac{\partial \mathbf{K}(\boldsymbol{\rho})}{\partial \rho_{e m}} \mathbf{W}(\boldsymbol{\rho})
\end{aligned}
$$

The matrix $\mathbf{W}(\rho)$ is the solution to the linear system of equations in (1) such that $\mathbf{W}(\rho)=\mathbf{K}(\rho)^{-1} \mathbf{F}$. The matrix $\mathbf{V}$ is obtained from the evaluation of $\mathbf{V}(\rho)=\mathbf{K}^{-T}(\rho) \mathbf{G}(\rho) \mathbf{W}(\rho)$, where the coefficient matrix $\mathbf{G}(\boldsymbol{\rho})$ has been defined in (2). Both $\mathbf{W}(\boldsymbol{\rho})$ and $\mathbf{V}(\rho)$ are determined only once and then reused in the evaluation of (12) for each of the design variables $\rho_{e m}$. Finally, inserting the result of (12) into (11) yields the gradients of the cross section stiffness matrix $\mathbf{K}_{s}$.

The gradients of the cross section mass matrix are obtained through differentiation of (3).

\subsection{Shear center}

The sensitivities of the shear center position with respect to the design variable $\rho_{e m}$ are obtained through differentiation of (10) to yield

$$
\begin{aligned}
& \frac{\partial x_{s}}{\partial \rho_{e m}}=-\frac{\partial F_{s, 62}}{\partial \rho_{e m}} \frac{1}{F_{s, 66}}+\frac{F_{s, 62}}{F_{s, 66}^{2}} \frac{\partial F_{s, 66}}{\partial \rho_{e m}}, \\
& \frac{\partial y_{s}}{\partial \rho_{e m}}=\frac{\partial F_{s, 61}}{\partial \rho_{e m}} \frac{1}{F_{s, 66}}-\frac{F_{s, 61}}{F_{s, 66}^{2}} \frac{\partial F_{s, 66}}{\partial \rho_{e m}}
\end{aligned}
$$

where the gradients $\partial F_{s, i j} / \partial \rho_{e m}$ refer to the entries of $\partial \mathbf{F}_{s} / \partial \rho_{e m}$ derived in (12).

\subsection{Eigenfrequencies}

The solution to the structural eigenvalue problem in (4) yields the eigenfrequencies and eigenvectors $\omega=\left\{\omega_{1}, \ldots, \omega_{n_{d}}\right\}$ and $\widehat{\mathbf{V}}=\left\{\widehat{\mathbf{v}}_{1}, \ldots, \widehat{\mathbf{v}}_{n_{d}}\right\}$, respectively. It is assumed that the eigenvectors are mass-normalized such that

$$
\widehat{\mathbf{v}}_{p}^{T} \widehat{\mathbf{M}}(\boldsymbol{\rho}) \widehat{\mathbf{v}}_{q}=\delta_{p q}, \forall p, q=1, \ldots, n_{d}
$$

where $n_{d}$ is the number of degrees of freedom, and $\delta_{p q}$ is the Kronecker delta such that $\delta_{p q}=1$ if $p=q$ and $\delta_{p q}=0$ otherwise. The gradient of a single eigenfrequency $\omega_{p}$ with respect to the design variable $\rho_{e m}$ is given by (cf. Seyranian et al. [37])

$$
\frac{\partial \omega_{p}^{2}(\boldsymbol{\rho})}{\rho_{e m}}=\widehat{\mathbf{v}}_{p}^{T}\left(\frac{\partial \widehat{\mathbf{K}}(\boldsymbol{\rho})}{\partial \rho_{e m}}-\omega_{p}^{2}(\boldsymbol{\rho}) \frac{\partial \widehat{\mathbf{M}}(\boldsymbol{\rho})}{\partial \rho_{e m}}\right) \widehat{\mathbf{v}}_{p}
$$

In the case of a multiple eigenfrequency, i.e., $\omega_{M}=\omega_{1}=$ $\ldots=\omega_{n_{\omega}}$, where the eigenfrequencies are numbered from 1 to $n_{\omega}$ for convenience and $n_{\omega}$ indicates the multiplicity of $\omega_{M}$, a different technique is applied. It can be shown that in this case, unlike the single eigenfrequency, the multiple eigenfrequency is not Fréchet differentiable. Seyranian et al. [37] instead derive an expression for the directional derivatives of multiple eigenvalues defined along any given vector $\Delta \rho$ of increments of the design variables $\rho$. A brief account of the procedure is described here. The first step consists of computing the entries of the generalized gradient vector $\boldsymbol{\Lambda}$ defined as

$$
\Lambda_{r s}=\widehat{\mathbf{v}}_{r}^{T}\left(\frac{\partial \widehat{\mathbf{K}}(\rho)}{\partial \rho_{e m}}-\omega_{M}^{2} \frac{\partial \widehat{\mathbf{M}}(\boldsymbol{\rho})}{\partial \rho_{e m}}\right) \widehat{\mathbf{v}}_{s}, r, s=1, \ldots, n_{\omega} .
$$


where $\widehat{\mathbf{v}}_{r}$ and $\widehat{\mathbf{v}}_{s}, r, s=1, \ldots, n_{\omega}$, are the eigenvectors associated with the multiple eigenfrequency $\omega_{M}$. The next step consists of determining the solution to the eigenvalue sub-problem

$$
(\boldsymbol{\Lambda}-\tilde{\lambda} \mathbf{I}) \tilde{\mathbf{v}}=\mathbf{0}
$$

which yields the eigenvalues $\tilde{\lambda}=\left\{\tilde{\lambda}_{1}, \ldots, \tilde{\lambda}_{n_{\omega}}\right\}$ and corresponding eigenvectors $\widetilde{\mathbf{V}}=\left\{\tilde{\mathbf{v}}_{1}, \ldots, \tilde{\mathbf{v}}_{n_{\omega}}\right\}$. It is assumed herein that the eigenvalues $\tilde{\lambda}$ are given in ascending order of magnitude such that $\tilde{\lambda}_{1}<\ldots<\tilde{\lambda}_{n_{\omega}}$. The eigenvalues $\tilde{\lambda}$ represent the directional derivatives $\partial \omega_{M}^{2}(\boldsymbol{\rho})$ of the multiple eigenfrequency $\omega_{M}$ such that

$$
\partial \omega_{M}^{2}(\boldsymbol{\rho})=\left\{\begin{array}{c}
\tilde{\lambda}_{1} \text { with eigenvector } \mathbf{v}_{1}=\sum_{q=1}^{n_{\omega}} \widehat{v}_{q} \tilde{v}_{1, q} \\
\vdots \\
\tilde{\lambda}_{n_{\omega}} \text { with eigenvector } \mathbf{v}_{n_{\omega}}=\sum_{q=1}^{n_{\omega}} \widehat{v}_{q} \tilde{v}_{n_{\omega}, q}
\end{array}\right.
$$

where $\tilde{v}_{n_{\omega}, q}$ is the entry $\left(n_{\omega}, q\right)$ of the eigenvector $\tilde{\mathbf{v}}$. Seyranian et al. [37] show based on numerical experiments that the directional derivatives $\partial \omega_{M}^{2}(\boldsymbol{\rho})$ match the gradients of the multiple eigenfrequency computed using finite differences.

The two techniques presented above are used for the evaluation of the beam eigenfrequencies throughout the optimization procedure. For a single eigenfrequency the sensitivities are given by the gradients $\partial \omega_{p}^{2}(\boldsymbol{\rho}) / \rho_{e m}$ in (13). An eigenfrequency $\omega_{M}$ is multiple when the relative difference between two or more consecutive eigenfrequencies in $\omega$ is below a predefined threshold. In this case the sensitivities of the multiple eigenvalue are given by the directional derivatives $\partial \omega_{M}^{2}(\rho)$ in (14).

Finally, the eigenfrequencies are included in the optimal design problem formulation through the KS function $\underline{\operatorname{KS}}(\rho)$ (see Section 3.2). The sensitivity of $\underline{\operatorname{KS}}(\rho)$ defined in (9) with respect to the design variables $\rho_{e m}$ for a set $\underline{\omega}$ of $n_{g}$ eigenfrequencies is given as

$$
\begin{aligned}
\frac{\partial \underline{\mathrm{KS}}(\boldsymbol{\rho})}{\partial \rho_{e m}} & =\frac{\partial \underline{\omega}_{n_{g}}(\boldsymbol{\rho})}{\partial \rho_{e m}}+ \\
& \frac{\sum_{g=1}^{n_{g}}\left(\frac{\partial \underline{\omega}_{g}(\boldsymbol{\rho})}{\partial \rho_{e m}}-\frac{\partial \underline{\omega}_{n_{g}}(\boldsymbol{\rho})}{\partial \rho_{e m}}\right) e^{-\beta_{s}\left(\underline{\omega}_{g}(\boldsymbol{\rho})-\underline{\omega}_{n_{g}}(\boldsymbol{\rho})\right)}}{\sum_{g=1}^{n_{g}} e^{-\beta_{s}\left(\underline{\omega}_{g}(\boldsymbol{\rho})-\underline{\omega}_{n g}(\boldsymbol{\rho})\right)}}
\end{aligned}
$$

The gradients of $\overline{\mathrm{KS}}(\rho)$ are easily obtained based on the expression above.

\section{Numerical experiments}

The optimal design framework described in the previous sections is employed in the optimal design of laminated composite beams. The geometrical properties of the beam are presented first. The mechanical properties of the considered materials are detailed next along with a description of the procedures entailed in the generation of the candidate materials. A brief description of the optimization strategy and details regarding the presentation of the results are subsequently presented. Finally, all the results are presented and discussed for each of the different problem formulations.

\subsection{Setup}

We consider the optimal design of a cantilever beam of constant cross section. The beam finite element model is composed of 16 three-node quadratic beam finite elements corresponding to 198 degrees of freedom. The coordinate system, finite element mesh and dimensions of the rectangular cross section or design domain are presented in Figure 2. The cross section is meshed using 2116 four-node isoparametric finite elements corresponding to 6627 degrees of freedom. The beam is $2.4 \mathrm{~m}$ wide, $2 \mathrm{~m}$ in height, and $20 \mathrm{~m}$ long. Two different materials have been considered - an orthotropic and an isotropic material - with the mechanical properties presented in Table 1. The mechanical properties of the orthotropic material are based on that of an E-glass reinforced Epoxy laminate (cf. Peters [38]). The mechanical properties of the isotropic material correspond to that of DIAB H100 PVC Core (DIAB [39]).

Nine candidate materials have been considered for all numerical experiments. The candidate materials are generated based on the material properties of the orthotropic layer and isotropic core material in Table 1. One of the candidates is the isotropic material itself. The remaining eight candidate materials are generated by rotation of the orthotropic layer. The orientation of the orthotropic layer in the cross section plane is specified based on the orientation $\alpha_{p}$ of the fiber plane and the orientation $\alpha_{f}$ of the fibers in the plane, cf. Figure 3. The eight candidate materials considered correspond to four different layer or fiber orientations $-0^{\circ}, 45^{\circ},-45^{\circ}$, and $90^{\circ}-$ stacked in either an horizontal $\left(0^{\circ}\right)$ or vertical $\left(90^{\circ}\right)$ fiber plane. Henceforth the nomenclature $\alpha_{f} @ \alpha_{p}$ will be used when referring to an orthotropic candidate material. For example, a layer oriented at $45^{\circ}$ stacked on a vertical plane $\left(90^{\circ}\right)$ is referred to as $45^{\circ} @ 90^{\circ}$ (cf. Figure 4).

\subsection{Optimization strategy}

The first step in the optimization is to solve the unpenalized problem corresponding to the case where $p=1$ in (7). At each of the subsequent steps, the penalty parameter $p$ is increased by $\Delta p$ until $p=p_{\text {max }}$. For all numerical experiments presented here it is assumed that $\Delta p=0.5$ and $p_{\max }=5$. Furthermore, for all cases, at the starting point all materials have the same volume fractions. The optimal design problems are solved using the sequential quadratic programming algorithm sNOPT by Gill et al. [32]. Each problem is solved until the major optimality tolerance is satisfied or the maximum number of major iterations is reached. The major optimality tolerance, like the major feasibility tolerance, is set to $1 \times 10^{-5}$ in SNOPT. The maximum number of major iterations is set to 500 in the first iteration and to 100 in the remaining. The remaining parameters are set to the default values. All numerical experiments are solved for 19044 
design variables associated with the nine candidate materials at each of the 2116 elements of the cross section. The filter radius is set to $f_{r}=0.15$ for all numerical experiments. Furthermore, the value of $\bar{w}$ indicates the fraction of the total weight which is orthotropic material. An equality constraint is used for the weight in order to avoid designs where only the isotropic material exists. Moreover, it is assumed that the eigenfrequency groups used in conjunction with the KS function are subsets of $\omega$ determined in (4). The eigenfrequency groups are denoted $\omega_{i, j}=\left\{\omega_{i}, \ldots, \omega_{j}\right\}$ where the subscripts $i$ and $j$ indicate the order of the lowest and higher eigenfrequencies in the group, respectively. The order number refers to the order placement in $\omega$. Only groups of consecutive eigenfrequencies have been considered such that $\omega_{i, j}$ includes the eigenfrequencies $i, j$, and all eigenfrequencies in between. The value of the parameter $\beta_{s}$ introduced in the definition of the KS function in (9) is set to unity for all cases. This ensures maximum numerical stability although the lower and upper bound of the eigenfrequency groups estimated by the KS function may be overly conservative. The sensitivity analysis technique for multiple eigenfrequencies presented in Section 4.4 is employed whenever the relative difference between two or more eigenfrequencies is below $1 \times 10^{-5}$.

\subsection{Presentation of the results}

All numerical examples considered concern the optimal design of prismatic beams, i.e., beam with constant cross section along its length. Hence for each case the figures with the results present only the cross section as this is sufficient to characterize the optimal structural topology and material distribution of the entire beam. Two figures of the optimized cross section are presented for each case indicating the fiber and fiber plane orientation, respectively. The topology of the cross section is visible in both. The fiber and fiber plane orientations are represented by lines at each element. The thickness and darkness of the line is weighted by the value of the filtered design variable. It is possible in this way to visualize the effect of the filter. Based on the orientation of the lines and resorting to Figure 4, it is possible to visualize the three-dimensional orientation of the fibers. The element is white if the material is isotropic. The plots refer to the unpenalized filtered design variables. Finally, the position of the cross section reference point, shear center, and mass center is indicated in these group of figures with a square, diamond, and triangle marker, respectively (cf. Figure 4).

\subsection{Results}

All numerical experiments considered in this paper are defined in Table 2. The corresponding objective function and constraint values for each of the numerical experiments are defined in Table 3. The optimized cross section topologies and material distribution are presented in Figures 5 and 7. The beam eigenmodes for a few relevant cases are presented in Figure 6 and 8. The eigenfrequencies, eigenmodes, constraint values, and number of objective function evaluations for each optimized design are presented in Table 4 . For all cases the weight constraint is satisfied and the shear center position constraint is active at the optimal design point. Furthermore, all values presented refer to the penalized case, i.e., $p=p_{\text {max }}$. The eigenmodes are identified based on its predominant motion. Bending eigenmodes with predominant displacements in the $x$ and $y$ direction are identified as $u_{x}$ and $u_{y}$, respectively. The torsional eigenmode associated with rotation of the cross section around the $z$ axis is identified as $\theta_{z}$. The tension eigenmode involving displacement of the cross sections along the $z$ axis is identified as $u_{z}$. The notation $u_{+x y}$ and $u_{-x y}$ is used for bending eigenmodes whose predominant displacements $x$ and $y$ are of similar magnitude. Eigenmodes indicated by $u_{+x y}$ and $u_{-x y}$ have a predominant displacement going through the first and third, and second and fourth quadrants of the $x y$ plane, respectively (see Figure 8).

The optimized beam cross section designs obtained for the maximization of the minimum eigenfrequency and maximization of the gap between eigenfrequencies - cases S1 and S2, respectively - are discussed first. The same formulations extended to include a constraint on the shear center position cases $\mathrm{S} 3$ and $\mathrm{S} 4$ - are discussed next.

\subsubsection{Results without constraint on the shear center position}

The S1 case dealing with the maximization of the lowest eigenfrequency and the cases S2-1, S2-2 and S2-3 addressing the maximization of the gap between different groups of eigenfrequencies (cf. Table 2 and 3) are discussed in this section. The resulting cross section topology and material distribution for cases S1 and S2 are presented in Figures 5(a-h). The lowest six eigenfrequencies and predominant displacement of the corresponding eigenvectors for each of the optimized beams are presented in Table 4.

For case S1, the results in Table 4 show that the magnitude of the two lowest eigenfrequencies are relatively similar and are associated with bending eigenmodes with predominant displacements in the $x$ and $y$ directions. The resulting box-like topology in Figures 5(a-b) maximizes the moment of inertia and therefore the bending stiffness of the beam in each of these directions. The fibers are aligned mostly along the beam length at $0^{\circ} @ 0^{\circ}$ and $0^{\circ} @ 90^{\circ}$ to maximize the bending stiffness. In the vertical or side faces, $\pm 45^{\circ} @ 90^{\circ}$ fibers are visible at the height of the horizontal neutral axis. These fibers resist the shear stresses induced by the transverse forces and contribute to an increase in shear stiffness in the $y$ direction and consequently of the lowest eigenfrequency $\omega_{1}$. This topology and material distribution is similar to that obtained by Blasques and Stolpe [11] for the minimum compliance optimization of a square beam subjected to a vertical transverse load.

For cases S2-1, S2-2 and S2-3 the eigenfrequencies and eigenmodes indicated in Table 4 show that all optimized designs maximize the gap between a bending and torsion eigenfrequency. The eigenmodes for the bending eigenfrequency $\omega_{4}$ and torsional eigenfrequency $\omega_{5}$ for case S2-3 are presented in Figure 6. Moreover, the progressive decrease in the magnitude of the lowest eigenfrequencies associated with bending eigenmodes suggests that the optimized beams are progressively more compliant in bending for case S2-1, S2-2 and S2-3, respectively. In fact, Figures $5(\mathrm{a}-\mathrm{h})$ show the transition from 
a tall and wide thin-walled beam stiff in bending - case S1 to a low radius, shaft-like, thick-walled cylinder with approximately the same torsional stiffness but relatively more compliant in bending - case S2-3. The predominant $0^{\circ} @ 0^{\circ}$ and $0^{\circ} @ 90^{\circ}$ laminates which in case S1 resist the normal stresses and maximize the bending stiffness are gradually replaced in the optimized designs of case S2-1 and S2-2 by $\pm 45^{\circ} @ 0^{\circ}$ and $\pm 45^{\circ} @ 90^{\circ}$ laminates. In the limit case S2-3 only interleaving layers of $\pm 45^{\circ} @ 0^{\circ}$ and $\pm 45^{\circ} @ 90^{\circ}$ fibers exist. This is such that the direction of maximum stiffness of the fibers is aligned at $\pm 45^{\circ}$ to resist the shear stresses and thus maximize the torsional stiffness. The decrease in torsional stiffness resulting from the reduction in cross section width and height is compensated by a redistribution of the material and an increase in wall thickness. As a result the torsional eigenfrequency of optimized designs S2-2 and S2-3 is close to that of S1.

\subsubsection{Results with constraint on shear center position}

Controlling the relative position of the shear, mass and reference centers it is possible, to a certain extent, to tailor the static and dynamic coupling between the bending and torsional motions. The preliminary results presented in this section illustrate the ability to do so within the proposed optimal design framework. Potential applications include aeroelastic tailoring of wind turbine blades for mitigation of dynamic instabilities, among other.

The S3 case addresses the maximization of the lowest eigenfrequency with a constraint on the shear center position whereas cases S4-1, S4-2 and S4-3 concern the maximization of the gap between different groups of eigenfrequencies with a constraint in the shear center position (cf. Table 3 ). The lowest six eigenfrequencies and predominant displacement of the corresponding eigenmodes, the weight, and the shear center position for each of the optimized designs are presented in Table 4. The resulting cross section topology and material distribution for cases S3 and S4 are presented in Figures 7(a-h).

The optimized cross section topology and material distribution for case S3 presented in Figures 7(a-b) are very similar to that of case $S 1$ in Figures 5(a-b). Furthermore, the magnitude of the lowest six eigenfrequencies and respective eigenmodes are also closely coinciding (cf. Table 4). A slight increase in the thickness of the right face of the beam section is observed in case $\mathrm{S} 3$ in order to satisfy the constraint in the position of the shear center,

The resulting optimized designs for cases S4-1, S4-2 and S43 are presented in Figures 7(c - h). The optimized designs S4-1 and S4-2, are similar to their counterparts S2-1 and S2-2 in Figures $5(\mathrm{c}-\mathrm{f})$ and, likewise, the designs are progressively more compliant in bending.

Unlike the remaining cases which dealt with the separation of a bending and torsional eigenfrequencies, in case S4-3 the optimization targets the maximization of the gap between two eigenfrequencies associated with bending eigenmodes. The resulting optimized design is a type of "I-beam" oriented diagonally with respect to the cross section coordinate system (see Figures $7(g-h))$. The resulting eigenmodes presented in Figure
8 show the direction of motion associated with the two eigenfrequencies being separated. The diagonal orientation is such that the height of the cross section and consequently the bending stiffness in that direction is maximized for the given design domain. The fibers align at $0^{\circ} @ 0^{\circ}$ and $0^{\circ} @ 90^{\circ}$ in the "flanges", or top left and bottom right regions of the design domain to maximize bending stiffness. In the central part, the shear stresses dominate and the laminates consisting of $\pm 45^{\circ} @ 0^{\circ}$ and $\pm 45^{\circ} @ 90^{\circ}$ fibers emerge to increase shear stiffness. The resulting optimized topology and material distribution is in fact similar to the results obtained by Blasques and Stolpe [11] for the minimum compliance optimization of a square beam subject to a diagonal transverse load.

\subsection{Discussion}

The framework initially presented by Blasques and Stolpe [11] is herein extended to include eigenfrequency constraints. The preliminary results presented in the previous sections indicate that the proposed framework is suitable for the simultaneous optimization of structural topology and material distribution in the design of beam cross sections with eigenfrequency constraints.

Regarding the optimization procedure, it can be seen that the number of objective function evaluations presented in Table 4 is relatively large when compared to typical topology optimization problems. This is mostly due to the relatively large number of steps used in the continuation approach and large number of allowed maximum major iterations at each of the steps. Numerical experience suggests that multi-material topology optimization problems are specially sensitive to the number of steps in the continuation approach. A larger number of smaller steps tends to generate designs with significantly improved performance and was therefore preferred. Nonetheless, note that the large number of objective function evaluations is possible only due to the high computational efficiency achieved by the the high-fidelity beam model used in this framework. The results further indicate that the KreisselmeierSteinhauser function used here to generate continuous and differentiable envelopes to approximate the maximum and minimum of groups of eigenfrequencies, is a possible alternative to the typical bound formulation (see, e.g., Du and Olhoff [4] and Bendsøe and Sigmund [20])). In the future, the two different formulations should be compared in terms of its convergence behaviour and the performance of the resulting optimized designs. At last, note that the interface to SNOPT does not provide the necessary information to plot the typical convergence plots. Furthermore, plotting the objective function versus the number of iterations neither reveals anything about the convergence rate nor provides a measure of closeness to a point satisfying the first order optimality conditions. The convergence plots are therefore omitted.

Future work will focus on truly exploiting the computational efficiency of the beam finite element model used in the proposed optimal design framework. The proposed framework opens the possibility for novel problem formulations including computationally intensive multiphysics and time dependent constraints associated with the global response of the beam. 
Moreover, a greater level of detail can be achieved by optimizing a larger number of cross sections along the length of the beam to obtain a detailed three-dimensional design of the beam structure. Potential applications include the threedimensional structural topology and material optimization of wind turbine blades with aeroelastic stability and fatigue constraints. Nonetheless, despite the focus on the design of laminated composite beams, the framework is sufficiently general and applicable to a broader class of problems dealing with the distribution of multiple materials to optimize the performance of beam-like structures. For example, the framework is well suited to topology optimization problems of beams with extrusion constraints. Typical approaches resort to computationally expensive three-dimensional solid finite element models and additional constraints on the design variables to ensure that the extrusion constraint is satisfied (see, e.g., Ishii and Aomura [40]). In this sense the approach proposed here has the potential to allow for a significant improvement in terms of computational efficiency.

\section{Conclusions}

A framework has been presented for the optimal design of beams with eigenfrequency constraints. The structural response of the beam is analyzed using beam finite elements. The cross section stiffness and mass properties are determined using a finite element based cross section analysis tool. The resulting beam model is able to account for effects stemming from material anisotropy and inhomogeneity in the analysis of beams with arbitrary section geometry.

The optimal design problem is formulated in a multi-material topology optimization context. An alternative approach is proposed to handle the eigenfrequencies constraints based on the Kreisselmeier-Steinhauser function.

Optimized cross section designs are presented for optimal design problems dealing with the maximization of the minimum eigenfrequency and the maximization of the gap between consecutive eigenfrequencies. Furthermore, results are also presented where the same problems are solved but the position of the shear center is constrained.

The numerical examples suggest that the devised optimal design framework is suitable for the simultaneous optimization of the cross section topology and material properties in the design of laminated composite beams with stiffness and eigenfrequency constraints. The next step consists of applying the presented methodology to the aeroelastic tailoring of laminated composite wind turbine blades.

\section{Acknowledgements}

The author would like to thank Senior Scientist Mathias Stolpe at Department of Wind Energy, Technical University of Denmark, for all fruitful discussions and suggestions.

\section{References}

1. Diaz A, Kikuchi N. Solutions to shape and topology eigenvalue optimization problems using a homogenization method. International Journal for Numerical Methods in Engineering 1992;35(7):1487-1502.

2. Ma Z, Kikuchi N, Hagiwara I. Structural topology and shape optimization for a frequency response problem. Computational mechanics 1993; 13:157-174.

3. Pedersen NL. Maximization of eigenvalues using topology optimization. Structural and multidisciplinary optimization 2000;20(1):2-11.

4. Du J, Olhoff N. Topological design of freely vibrating continuum structures for maximum values of simple and multiple eigenfrequencies and frequency gaps. Structural and Multidisciplinary Optimization 2007; 34(2):91-110.

5. Luo J, Gea H. A systematic topology optimization approach for optimal stiffener design. Structural and Multidisciplinary Optimization 1998; 16:280-288.

6. Gea H, Luo J. Automated Optimal Stiffener Pattern Design. Journal of Structural Mechanics 1999;27(3):275-292.

7. Stegmann J, Lund E. Discrete material optimization of general composite shell structures. International Journal for Numerical Methods in Engineering 2005;62(14):2009-2027.

8. Pedersen NL. On design of fiber-nets and orientation for eigenfrequency optimization of plates. Computational Mechanics 2005;39(1):1-13.

9. Olhoff N. Optimization of vibrating beams with respect to higher order natural frequencies. Journal of Structural Mechanics 1976;4:87-122.

10. Bendsøe MP, Olhoff N. A method of design against vibration resonance of beams and shafts. Optimal Control Applications and Methods 1985; 6(3):191-200

11. Blasques JP, Stolpe M. Multi-material topology optimization of laminated composite beam cross sections. Composite Structures 2012; 94(11):3278-3289.

12. Larsen T, Hansen A. How 2 HAWC2, the user's manual. Denmark. Forskningscenter Risoe. Risoe-R. Ris $\varnothing$ National Laboratory; 2007. ISBN 978-87-550-3583-6.

13. Blasques JP, Lazarov B. BECAS - A beam cross section analysis tool for anisotropic and inhomogeneous sections of arbitrary geometry. Ris $\varnothing-\mathrm{R}$ 1785 Technical Report; 2011.

14. Giavotto V, Borri M, Mantegazza P, Ghiringhelli G, Carmaschi V, Maffioli $\mathrm{G}$, et al. Anisotropic beam theory and applications. Computers $\mathcal{F}$ Structures 1983;16(1-4):403-413.

15. Jung S, Nagaraj V, Chopra I, Others . Assessment of composite rotor blade modeling techniques. Journal of the American Helicopter Society 1999;44(3): 188 .

16. Volovoi V, Hodges D, Cesnik C, Popescu B. Assessment of beam modeling methods for rotor blade applications. Mathematical and Computer Modelling 2001;33(10-11):1099-1112.

17. Hodges DH. Nonlinear composite beam theory; vol. 3. American Institute of Aeronautics and Astronautics; 2006.

18. Hvejsel CF, Lund E. Material interpolation schemes for unified topology and multi-material optimization. Structural and Multidisciplinary Optimization 2011;43:811-825.

19. Hvejsel CF, Lund E, Stolpe M. Optimization strategies for discrete multimaterial stiffness optimization. Structural and Multidisciplinary Optimization 2011;44:149-163.

20. Bendsøe MP, Sigmund O. Topology optimization: theory, methods, and applications. Springer Verlag; 2nd ed.; 2003. ISBN 3540429921.

21. Bendsøe MP, Kikuchi N. Generating optimal topologies in structural design using a homogenization method. Computer Methods in Applied Mechanics and Engineering 1988;71:197-224.

22. Rozvany GI, Zhou M. The COC algorithm, Part I: Cross-section optimization or sizing. Computer Methods in Applied Mechanics and Engineering 1991;89:281-308.

23. Bruns T, Tortorelli D. Topology optimization of non-linear elastic structures and compliant mechanisms. Computer Methods in Applied Mechanics and Engineering 2001;190(26-27):3443-3459.

24. Lund E, Stegmann J. On structural optimization of composite shell structures using a discrete constitutive parametrization. Wind Energy 2005; 8(1):109-124.

25. Kreisselmeier G, Steinhauser R. Systematic control design by optimizing a vector performance index. In: International Federation of Active Con- 
trols Symposium on Computer-Aided Design of Control Systems. Zurich; 1979, .

26. Raspanti C, Bandoni J, Biegler L. New strategies for flexibility analysis and design under uncertainty. Computers $\mathcal{E}$ Chemical Engineering 2000; 24(9-10):2193-2209.

27. Martins J, Alonso J, Reuther J. A coupled-adjoint sensitivity analysis method for high-fidelity aero-structural design. Optimization and Engineering 2005;6(1):33-62.

28. Maute K, Nikbay M, Farhat C. Sensitivity analysis and design optimization of three-dimensional non-linear aeroelastic systems by the adjoint method. International Journal for Numerical Methods in Engineering 2003;56(6):911-933.

29. Bathe KJ. Finite Element Procedures. Englewood Cliffs - New Jersey: Prentice Hall; 1996.

30. Sigmund O, Petersson J. Numerical instabilities in topology optimization: A survey on procedures dealing with checkerboards, mesh-dependencies and local minima. Structural Optimization 1998;16(1):68-75.

31. Borrvall T, Petersson J. Topology optimization using regularized intermediate density control. Computer Methods in Applied Mechanics and Engineering 2001;190(37-38):4911-4928.

32. Gill P, Murray W, Saunders M. SNOPT: An SQP algorithm for large scale constrained optimization. SIAM Journal on Optimization 2002; 121(4):979-1006.

33. Sørensen SN, Lund E. Topology and thickness optimization of laminated composites including manufacturing constraints. Structural and Multidisciplinary Optimization 2013;48(2):249-265.

34. Sigmund O. Morphology-based black and white filters for topology optimization. Structural and Multidisciplinary Optimization 2007;33(45):401-424.

35. Bourdin B. Filters in topology optimization. International Journal for Numerical Methods in Engineering 2001;50(9):2143-2158.

36. Hansen M. Aeroelastic instability problems for wind turbines. Wind Energy 2007;10(6):551-577.

37. Seyranian AP, Lund E, Olhoff N. Multiple eigenvalues in structural optimization problems. Structural Optimization 1994;8(4):207-227.

38. Peters S. Handbook of composites. London: Chapman and Hall; 2nd ed.; 1998

39. DIAB Group . Divinycell H100 Data Sheet. 2011. URL www. diabgroup. com.

40. Ishii K, Aomura S. Topology optimization for the extruded three dimensional structure with constant cross section. JSME International Journal Series A Solid Mechanics and Material Engineering 2004;47(2):198-206. 
7. Tables 
Table 1: Material properties for orthotropic material (scaled values for E-glass reinforced Epoxy laminate according to Peters [38]), and isotropic material (scaled values for DIAB H100 PVC Core, cf. DIAB DIAB Group [39]) (Table from Blasques and Stolpe [11]).

\begin{tabular}{crlrl}
\hline Material & \multicolumn{2}{c}{ Orthotropic } & \multicolumn{2}{c}{ Isotropic } \\
\hline$E_{11}$ & 480 & $\mathrm{GPa}$ & 0.130 & $\mathrm{GPa}$ \\
$E_{22}=E_{33}$ & 120 & $\mathrm{GPa}$ & 0.130 & $\mathrm{GPa}$ \\
$G_{12}=G_{13}$ & 60 & $\mathrm{GPa}$ & 0.035 & $\mathrm{GPa}$ \\
$G_{23}$ & 50 & $\mathrm{GPa}$ & 0.035 & $\mathrm{GPa}$ \\
$v_{12}=v_{13}$ & 0.19 & - & 0.35 & - \\
$v_{23}$ & 0.26 & - & 0.35 & - \\
$\bar{Q}$ & 1.78 & $10^{3} \mathrm{~kg} / \mathrm{m}^{3}$ & 0.1 & $10^{3} \mathrm{~kg} / \mathrm{m}^{3}$ \\
\hline
\end{tabular}

Table 2: Catalogue of numerical experiments combining the different problem formulations.

\begin{tabular}{|c|c|}
\hline Ref. & Problem formulation \\
\hline $\mathrm{S} 1$ & $\begin{array}{c}\text { Max. min. eigenfrequency } \\
\text { with a weight constraint (P1-3) }\end{array}$ \\
\hline S2-1 & $\begin{array}{l}\text { Max. gap between two eigenfrequencies with } \\
\text { constraints on weight }(\mathrm{P} 1-2)\end{array}$ \\
\hline S2-2 & $\begin{array}{l}\text { Max. gap between two eigenfrequencies with } \\
\text { constraints on weight }(\mathrm{P} 1-2)\end{array}$ \\
\hline S2-3 & $\begin{array}{l}\text { Max. gap between two eigenfrequencies with } \\
\text { constraints on weight }(\mathrm{P} 1-2)\end{array}$ \\
\hline S3 & $\begin{array}{l}\text { Max. min. eigenfrequencies with constraints } \\
\text { on weight and shear center position (P1-4) }\end{array}$ \\
\hline S4-1 & $\begin{array}{l}\text { Max. gap between eigenfreqs. with constraints } \\
\text { on weight and shear center position (P1-1) }\end{array}$ \\
\hline S4-2 & $\begin{array}{l}\text { Max. gap between eigenfreqs. with constraints } \\
\text { on weight and shear center position (P1-1) }\end{array}$ \\
\hline S4-3 & $\begin{array}{l}\text { Max. gap between eigenfreqs. with constraints } \\
\text { on weight and shear center position (P1-1) }\end{array}$ \\
\hline
\end{tabular}

Table 3: Details for all numerical experiments (cf. Table 2). The first column indicates the objective function. The eigenfrequency groups are denoted $\omega_{i, j}=\left\{\omega_{i}, \ldots, \omega_{j}\right\}$ where the subscripts $i$ and $j$ indicate the order of the lowest and highest eigenfrequencies in the group, respectively. The second column indicates the value of the equality constraint on the weight where $\bar{w}$ refers to the ratio of orthotropic material. The last column indicates the constraints on the shear center position $\mathbf{s}_{c}=\left(x_{s}, y_{s}\right)$.

\begin{tabular}{cccc}
\hline Ref. & Obj. func. & $\bar{w}$ & $\overline{\mathbf{s}}_{c}$ \\
\hline $\mathrm{S} 1$ & $\underline{\mathrm{KS}}\left(\omega_{1,5}\right)$ & $3 / 5$ & - \\
$\mathrm{S} 2-1$ & $\underline{\mathrm{KS}}\left(\omega_{3,4}\right)-\overline{\mathrm{KS}}\left(\omega_{1,2}\right)$ & $3 / 5$ & - \\
$\mathrm{S} 2-2$ & $\underline{\mathrm{KS}}\left(\omega_{4,6}\right)-\overline{\mathrm{KS}}\left(\omega_{1,3}\right)$ & $3 / 5$ & - \\
$\mathrm{S} 2-3$ & $\underline{\mathrm{KS}}\left(\omega_{5,7}\right)-\overline{\mathrm{KS}}\left(\omega_{2,4}\right)$ & $3 / 5$ & - \\
$\mathrm{S} 3$ & $\underline{\mathrm{KS}}\left(\omega_{1,5}\right)$ & $3 / 5$ & $x_{s}=-0.2, y_{s}=0$ \\
$\mathrm{~S} 4-1$ & $\underline{\mathrm{KS}}\left(\omega_{1,2}\right)-\overline{\mathrm{KS}}\left(\omega_{3,4}\right)$ & $3 / 5$ & $x_{s}=-0.2, y_{s}=0$ \\
$\mathrm{~S} 4-2$ & $\underline{\mathrm{KS}}\left(\omega_{1,3}\right)-\overline{\mathrm{KS}}\left(\omega_{4,6}\right)$ & $3 / 5$ & $x_{s}=-0.2, y_{s}=0$ \\
$\mathrm{~S} 4-3$ & $\underline{\mathrm{KS}}\left(\omega_{2,4}\right)-\overline{\mathrm{KS}}\left(\omega_{5,7}\right)$ & $3 / 5$ & $x_{s}=-0.2, y_{s}=0$ \\
\hline
\end{tabular}

Table 4: Summary of numerical results for optimized designs for cases S1 through S4 and all sub-cases therein (cf. Table 3). The first six rows indicated the lowest six eigenfrequencies for the optimized designs (the lines are placed between the eigenfrequencies that have been separated). The letters in brackets indicate the predominant motion of the corresponding eigenmodes $\left(u_{x}\right.$, $u_{y}$, and $u_{z}$, indicate displacements in the direction $x, y$, and $z$, respectively; $\theta_{z}$ indicates torsion; $u_{+x y}$ and $u_{-x y}$ indicates a predominant displacement motion between the first and third, and second and fourth quadrants of the $x y$ plane, respectively). The resulting weight $(w)$, shear center position $\left(\mathbf{s}_{c}=\left(x_{s}, y_{s}\right)\right)$, and number of objective function evaluations are presented in the following rows, respectively. The eigenfrequency values and the shear center position values are obtained with the penalized filtered densities, i.e., $p=p_{\max }=5$.

\begin{tabular}{|c|c|c|c|c|}
\hline & $\mathrm{S} 1$ & S2-1 & S2-2 & $\mathrm{S} 2-3$ \\
\hline$\omega_{1}$ & $5.9\left(u_{y}\right)$ & $4.2\left(u_{x}\right)$ & $0.7\left(u_{y}\right)$ & $0.5\left(u_{y}\right)$ \\
\hline$\omega_{2}$ & $7.3\left(u_{x}\right)$ & $4.4\left(u_{y}\right)$ & $2.9\left(u_{x}\right)$ & $0.5\left(u_{x}\right)$ \\
\hline$\omega_{3}$ & $69.5\left(\theta_{z}\right)$ & $99.0\left(\theta_{z}\right)$ & $23.5\left(u_{y}\right)$ & $18.1\left(u_{y}\right)$ \\
\hline$\omega_{4}$ & $99.7\left(u_{y}\right)$ & $111.0\left(u_{x}\right)$ & $71.7\left(\theta_{z}\right)$ & $19.3\left(u_{x}\right)$ \\
\hline$\omega_{5}$ & $170.0\left(u_{x}\right)$ & $114.0\left(u_{y}\right)$ & $85.7\left(u_{x}\right)$ & $76.0\left(\theta_{z}\right)$ \\
\hline$\omega_{6}$ & $439.9\left(u_{y}\right)$ & $579.9\left(u_{z}\right)$ & $154.5\left(u_{y}\right)$ & $128.9\left(u_{y}\right)$ \\
\hline$w$ & $3 / 5$ & $3 / 5$ & $3 / 5$ & $3 / 5$ \\
\hline \multirow[t]{2}{*}{ Fun. Eval. } & 546 & 1736 & 2336 & 2269 \\
\hline & S3 & S4-1 & $\mathrm{S} 4-2$ & $\mathrm{~S} 4-3$ \\
\hline$\omega_{1}$ & $5.9\left(u_{y}\right)$ & $3.7\left(u_{x}\right)$ & $1.2\left(u_{-x y}\right)$ & $0.7\left(u_{+x y}\right)$ \\
\hline$\omega_{2}$ & $7.5\left(u_{x}\right)$ & $3.9\left(u_{y}\right)$ & $3.6\left(u_{+x y}\right)$ & $7.9\left(u_{-x y}\right)$ \\
\hline$\omega_{3}$ & $66.3\left(\theta_{z}\right)$ & $82.8\left(\theta_{z}\right)$ & $38.4\left(u_{-x y}\right)$ & $20.8\left(\theta_{z}\right)$ \\
\hline$\omega_{4}$ & $96.2\left(u_{y}\right)$ & $98.5\left(u_{x}\right)$ & $100.6\left(\theta_{z}\right)$ & $25.5\left(u_{+x y}\right)$ \\
\hline$\omega_{5}$ & $176.1\left(u_{x}\right)$ & $100.0\left(u_{y}\right)$ & $105.1\left(u_{+x y}\right)$ & $159.9\left(u_{-x y}\right)$ \\
\hline$\omega_{6}$ & $413.7\left(u_{y}\right)$ & $495.8\left(u_{z}\right)$ & $244.2\left(u_{-x y}\right)$ & $161.0\left(u_{+x y}\right)$ \\
\hline$w$ & $3 / 5$ & $3 / 5$ & $3 / 5$ & $3 / 5$ \\
\hline$x_{s}$ & -0.2 & -0.2 & -0.2 & -0.2 \\
\hline$y_{s}$ & 0.0 & 0.0 & 0.0 & 0.0 \\
\hline Fun. Eval. & 760 & 1682 & 2213 & 2869 \\
\hline
\end{tabular}


8. Figures 


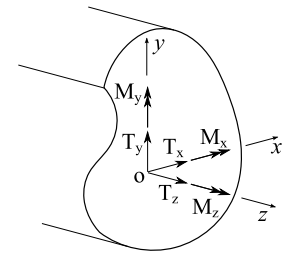

(a) Forces and moments (b) Strains and curvatures
Figure 1: Cross section coordinate system, foces and moments (a), and corresponding strains and curvatures (b).

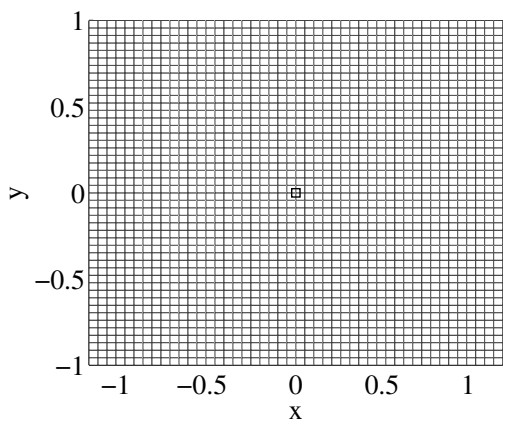

Figure 2: Cross section coordinate system and finite element mesh. The cross section is meshed using 2116 four-node isoparametric finite elements corresponding to 6627 degrees of freedom. The square marker indicates the position of the cross section reference point or beam node.

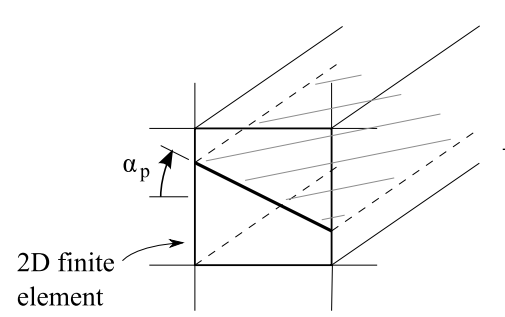

Fiber plane orientation

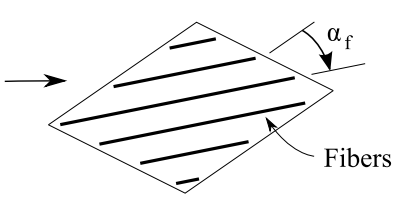

Fiber orientation
Figure 3: Three-dimensional rotation of fiber plane and fiber orientation in the cross section mesh. The fiber plane orientation is defined by the angle $\alpha_{p}$ while the orientation of the fibers in the fiber plane are defined by the angle $\alpha_{f}$. (Figure from Blasques and Stolpe [11])

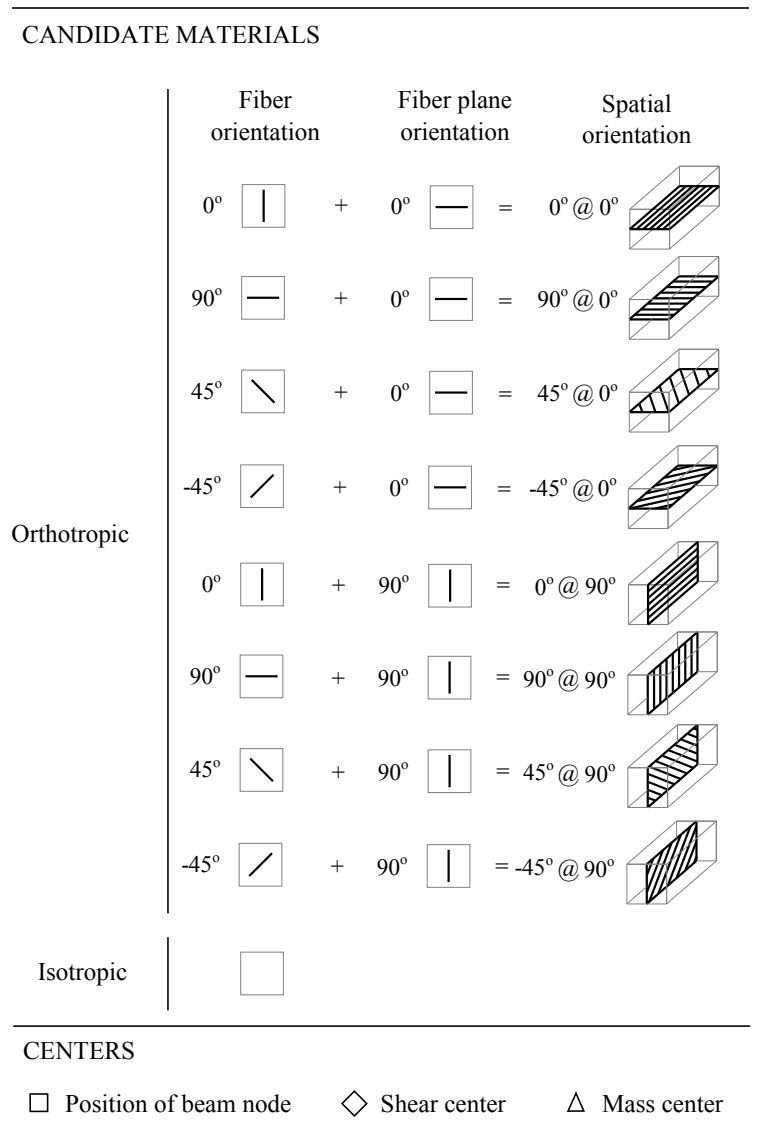

Figure 4: Legend for the figures depicting the solutions. Visualization of the spatial orientation of the fibers at each element based on the resulting fiber and fiber plane orientations, for each of the candidate orthotropic materials. Description of the markers used to define the cross section reference point or beam node (square), shear center (diamond), and mass center (triangle). (Figure from Blasques and Stolpe [11]) 


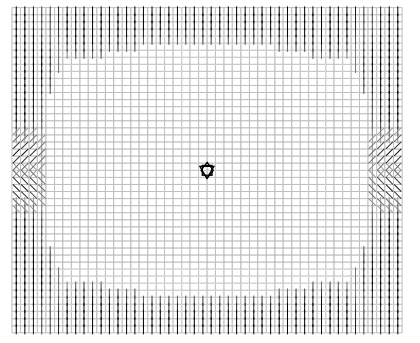

(a) S1 Fiber angles

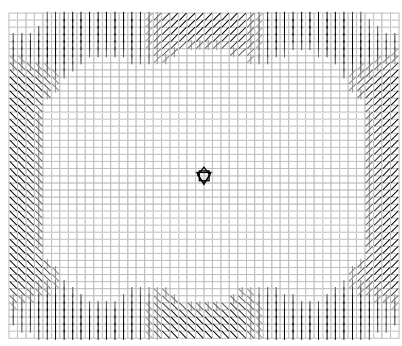

(c) S2-1 Fiber angles

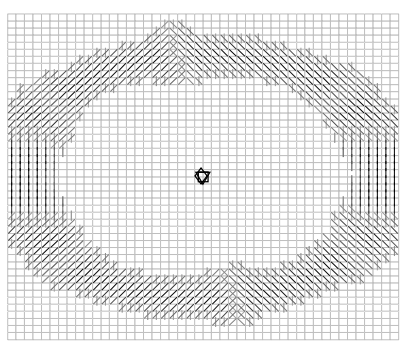

(e) S2-2 Fiber angles

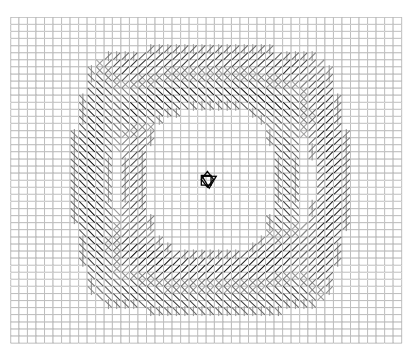

(g) S2-3 Fiber angles

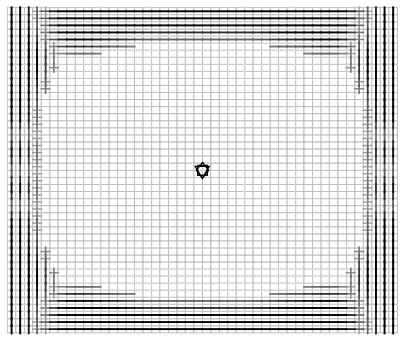

(b) S1 Fiber plane angles

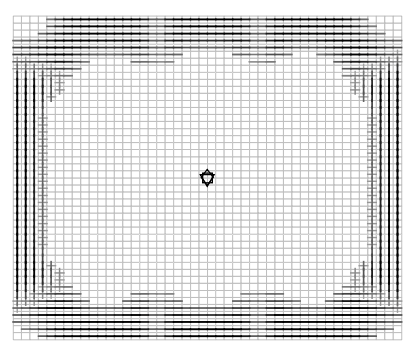

(d) S2-1 Fiber plane angles

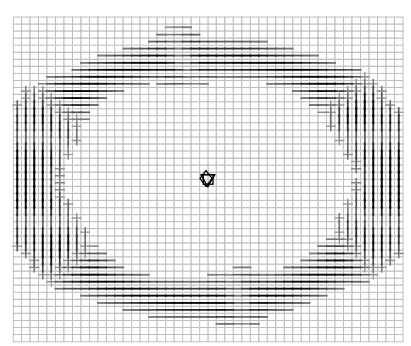

(f) S2-2 Fiber plane angles

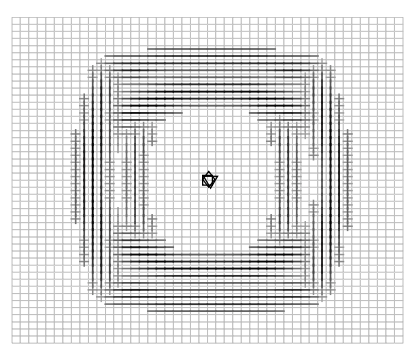

(h) S2-3 Fiber plane angles

Figure 5: Optimal cross section topology and material distribution for cases S1, S2-1, S2-2, and S2-3 (cf. Tables 2 and 3). Case S1 is the solution to the maximization of the minimum eigenfrequecy problem with a weight constraint (P1-3). The remaining cases S2-1, S2-2, and S2-3 are the solutions to the maximization of the gap between two eigenfrequencies with weight constraints (P1-2). The legend to the figures is described in Figure 4.

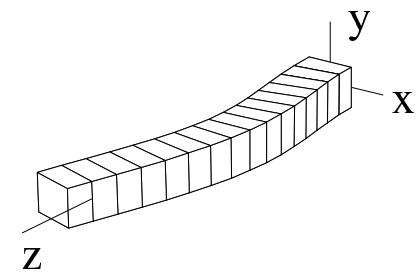

(a) $\mathrm{S} 2-3, \omega_{4}\left(u_{x}\right)=19.3$

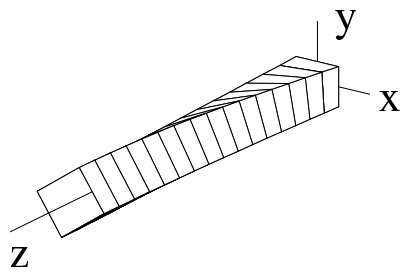

(b) $\mathrm{S} 2-3, \omega_{5}\left(\theta_{z}\right)=76.0$

Figure 6: Beam eigenmodes for the fourth and fifth eigenfrequency (cf. Table 4) of optimized cantilever beam for case S2-3 (cf. Table 3). The corresponding optimized cross section topology and material distribution for this case is presented in Figure $5(\mathrm{~g}-\mathrm{h})$. 


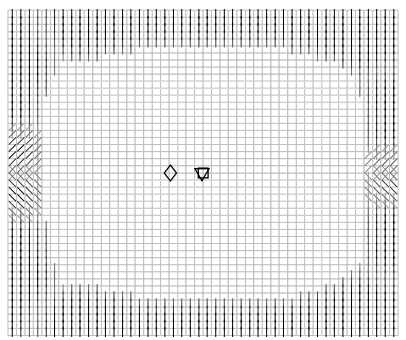

(a) S3 Fiber angles

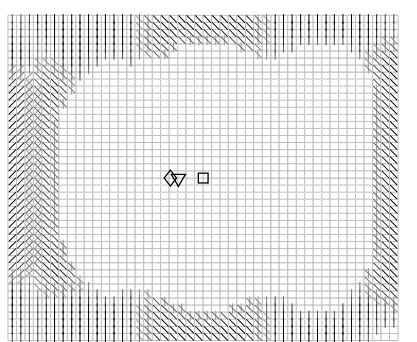

(c) S4-1 Fiber angles

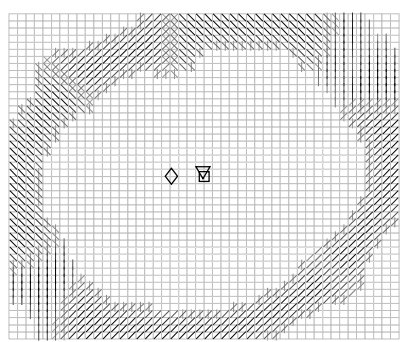

(e) S4-2 Fiber angles

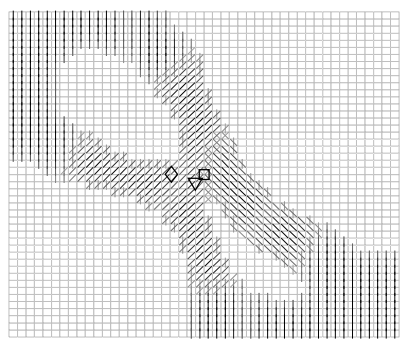

(g) S4-3 Fiber angles

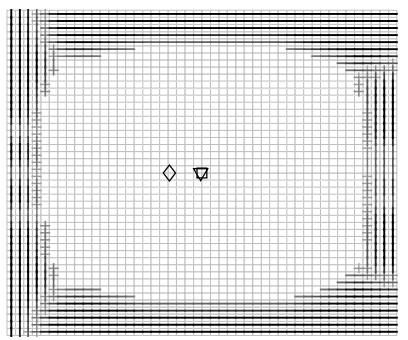

(b) S3 Fiber plane angles

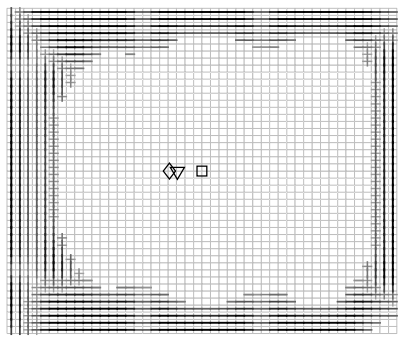

(d) S4-1 Fiber plane angles

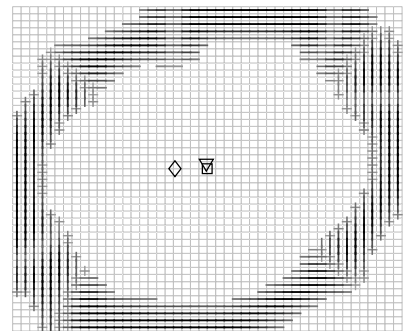

(f) S4-2 Fiber plane angles

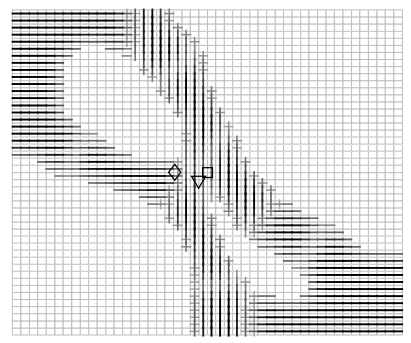

(h) S4-3 Fiber plane angles
Figure 7: Optimal cross section topology and material distribution for cases S3, S4-1, S4-2, and S4-3 (cf. Tables 2 and 3). Case S3 is the solution to the maximization of the minimum eigenfrequecy problem with constraints on weight and shear center position (P1-4). The remaining cases S4-1, S4-2, and S4-3 are the solutions to the maximization of the gap between two eigenfrequencies with constraints on weight and shear center position (P1-1). The legend to the figures is described in Figure 4.
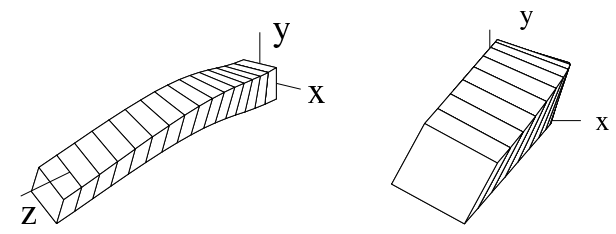

(a) S4-3, $\omega_{4}\left(u_{+x y}\right)=25.5$
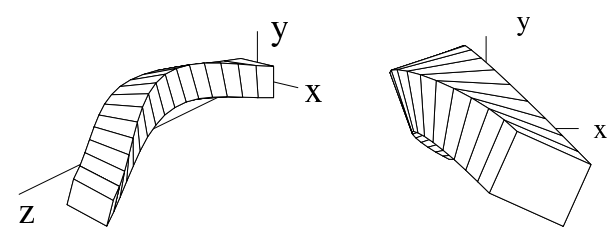

(b) $\mathrm{S} 4-3, \omega_{5}\left(u_{-x y}\right)=159.9$

Figure 8: Schematic view of the eigenmodes for the fourth and fifth eigenfrequency (cf. Table 4) of optimized beam for case S4-3 (cf. Table 3) - perspective (left) and front (right) view. The corresponding optimized cross section topology and material distribution for this case is presented in Figure 7 (g-h). 